\title{
L'avvio della ricerca empirica in campo educativo in Italia: il contributo di Calonghi e Visalberghi
}

\section{Giuseppe Zanniello}

Università degli Studi di Palermo, Dipartimento di Psicologia

doi: 10.7358/ecps-2014-009-zann

giuseppe.zanniello@unipa.it

\section{THE START OF EMPIRICAL RESEARCH IN EDUCATION \\ IN ITALY: THE CONTRIBUTION OF CALONGHI \\ AND VISALBERGHI}

\section{Abstract}

While the dominant philosophy in Italy at the end of World War II was not favourable to pedagogical experimentation, some initiatives aiming to innovate - in the activist sense contents and methods of school teaching represented the remote causes of the start of educational studies with the positive method. These initiatives facilitated the action of empirical research pioneers in the field of education, which developed in Italy in the 1950s for three main reasons: (1) the influence of experiments conducted in U.S. schools, (2) the spreading of Dewey's thought, (3) the impetus given by the Institute of Pedagogy of the Salesians to the study of educational sciences. Calonghi and Visalberghi were the first to promote and organize two lines of empirical research on educational facts which contributed to the full scientific recognition of Experimental Pedagogy and Docimology in the mid-1970s. In order to avoid technicalities, the researchers who use the experimental method feel the need to frame their specialized studies within a vast pedagogical horizon.

Keywords: Calonghi, Empirical research, Experiential pedagogy, Experimental pedagogy, Visalberghi. 


\section{IL SIGNIFICATO DELLA RICERCA EMPIRICA SUI FATTI EDUCATIVI}

Qual è il vero significato della ricerca empirica? La risposta oggi è facile: gli studi pedagogici che vogliano ottenere un riconoscimento scientifico hanno bisogno di una base fattuale, devono partire dall'esperienza e ad essa ritornare dopo un'attenta riflessione; la ricerca empirica è indispensabile per la costruzione del sapere pedagogico. Tuttavia tale chiarezza di idee non esisteva sessanta anni fa tra i pedagogisti italiani.

Laeng (1997, pp. 139-140) osserva giustamente che l'espressione ricerca empirica "ha ricevuto talvolta un'accezione riduttiva, come se alludesse a una raccolta saltuaria, occasionale e poco sistematica, affidata alla memoria soggettiva piuttosto che a protocolli e registrazioni rigorose. Va appena detto che questa opinione riduttiva ha avuto purtroppo molte conferme nel grado approssimativo delle generalizzazioni pedagogiche, spesso ridotte a superficiali ovvietà o affidate alla 'buona volontà' degli educatori e alla divulgazione dei letterati. Ma così non dovrebbe essere, qualora si intendesse l'esperienza come raccolta di dati guidata e giudicata dalla ragione. La componente empirica diventa allora metodo osservativo-sperimentale».

Non c'è dubbio che in ogni atto educativo c'è qualcosa di unico e di irripetibile; ma è anche difficile negare che ci siano alcune somiglianze pedagogiche tra diversi momenti, occasioni e situazioni educative scientificamente studiate. La ricerca empirica cerca proprio di scoprire, analizzare e classificare le somiglianze che si notano nei fenomeni educativi tentando di individuare delle tipologie ricorrenti, di riflettere su di esse e di enunciare dei principi orientativi dell'azione educativa in situazioni simili a quelle studiate.

Quando si esaminano i prodotti della ricerca empirica in campo educativo si evince che essi riguardano più la Didattica che la Pedagogia. Ciò non sorprende perché è più facile giustificare con dati di fatto la validità di un metodo di insegnamento-apprendimento che non un metodo per l'educazione alla libertà e alla responsabilità dei giovani. La Didattica, intesa come scienza che studia il processo di insegnamento-apprendimento, si costruisce a partire dalla riflessione sulla pratica professionale di chi insegna e sulla sua ipotizzata relazione con l'apprendimento degli alunni. Mentre la riflessione esige nel ricercatore la condivisione di una concezione dell'uomo e della sua educazione, la raccolta fedele delle pratiche di insegnamento e dei risultati dell'apprendimento richiede l'uso da parte sua del metodo empirico. La riflessione pedagogica produce risultati scientificamente validi se è svolta su dati ricavati fedelmente dalla realtà educativa per come essa è, con metodi, tecniche e strumenti adeguati.

Il cammino che ha portato al riconoscimento accademico della ricerca empirica in Italia non è stato facile; saranno descritte di seguito alcune ca- 
ratteristiche della fase iniziale di questa tradizione di ricerca pedagogica nel nostro Paese. La ricostruzione storica non è solo un omaggio a coloro che per primi seppero intravedere nuove piste di ricerca per venire incontro alle mutate esigenze della scuola, degli insegnanti e degli alunni. La riflessione sulle origini di un movimento di pensiero, che continua a produrre apprezzabili risultati scientifici, specialmente in campo didattico, grazie al rigore dell'apparato metodologico impiegato, orienta la proiezione delle indagini verso il futuro; la proposta educativa infatti si dovrà sempre adeguare alle cangianti condizioni in cui vivono i giovani.

Nel volgere lo sguardo indietro, nel riflettere sul cammino percorso si intende offrire un contributo per tirar fuori la ricerca didattica dalle secche del tecnicismo, affinché essa sappia rispondere adeguatamente alle pressanti richieste di senso che provengono da quanti sono quotidianamente impegnati nel lavoro educativo in favore degli alunni. Il ritorno alle radici, vale a dire alle motivazioni ideali che sorressero gli iniziatori, in Italia, della ricerca didattica basata sull'aderenza all'esperienza degli educatori e sull'impiego di un apparato metodologico di tipo sperimentale, potrebbe offrire un contributo in tal senso.

\section{CRiteri Di DATAZione}

La ricerca empirica in campo educativo, per come è stata definita sopra, muove con continuità i primi passi in Italia all'inizio degli anni cinquanta del ventesimo secolo. Nel nostro Paese, il periodo pionieristico si è concluso negli anni sessanta piuttosto che in quelli sessanta, perché nel decennio 1960-70 da noi non ci fu ancora l'esplosione della Pedagogia sperimentale, come invece avvenne negli altri Paesi dell'Europa occidentale (De Landsheere, 1988, p. 107). In Italia, dal punto di vista quantitativo, negli anni sessanta ci fu un significativo sviluppo di indagini che denunciarono la selezione scolastica come strumento di selezione sociale, individuarono le cause socio-economiche dell'insuccesso scolastico, evidenziarono i pregiudizi di molti insegnanti nei confronti degli alunni culturalmente svantaggiati. Ma, bisogna riconoscerlo, allora erano davvero in pochi a svolgere delle ricerche scientifiche per saggiare la capacità di nuove metodologie educative di ridurre le ingiustizie così spesso denunciate. Si potrebbe affermare che negli anni sessanta si coltivarono le motivazioni ideali per realizzare, con una scuola rinnovata nei contenuti e nei metodi didattici, la tanto auspicata equità sociale e per l'acquisizione da parte di un significativo numero di ricercatori, dei metodi e delle tecniche della ricerca empirica a sostegno dell'innovazione educativa. 
In considerazione del fatto che la ricerca empirica degli anni cinquanta nasce su presupposti epistemologici diversi da quelli di inizio secolo, sono tralasciati in questo intervento i suoi prodromi nel ventennio 1895-1915, quando il positivismo filosofico faceva sentire il suo influsso sulla pedagogia; poi, come è noto, la tradizione di ricerca empirica si interruppe con la prima guerra mondiale e non riprese finché il clima culturale in Italia fu dominato dall'idealismo gentiliano. In ogni caso occorre considerare che la ripresa della ricerca empirica in campo educativo, intorno al 1950, avvenne con riferimenti teorici caratterizzati dalla discontinuità con quanto era avvenuto prima delle due guerre mondiali.

Pertanto, dopo la breve fase di inizio secolo, la ricerca empirica in campo educativo in Italia inizia sostanzialmente con gli anni cinquanta; anche se nel corso del XX secolo, come si legge nei testi di storia dell'educazione, c'erano state delle validissime innovazioni scolastiche - documentate con diari, relazioni e descrizioni dei metodi e delle tecniche impiegate -, era mancato nei loro promotori l'intento di scegliere dei campioni rappresentativi, di costruire disegni sperimentali, di tenere sotto controllo le variabili e di verificare i risultati delle innovazioni introdotte con strumenti validi e affidabili.

Il periodo preso in considerazione inizia con la pubblicazione dei primi studi italiani su John Dewey, che nei sessanta anni precedenti era stato l'ispiratore della sperimentazione scolastica in USA, e con le prime ricerche sperimentali di Calonghi e di Visalberghi. Quando nel 1950 Corallo pubblicò il volume La pedagogia di Giovanni Dewey, seguito l'anno successivo dal volume J. Dewey, di Visalberghi in Italia erano stati tradotti pochissimi scritti del pedagogista americano. Lanno di conclusione del periodo pioneristico è più opinabile perché non è legato a date di pubblicazioni, ma dipende da un giudizio soggettivo circa il momento in cui avvenne il riconoscimento sociale della dignità scientifica della ricerca sperimentale sui fatti educativi. Tuttavia, se si volesse assumere un anno «ideale» di riferimento, esso potrebbe essere il 1974, vale a dire quello dell'emanazione del D.P.R. n. 419 sulla sperimentazione scolastica. In quella occasione infatti furono istituiti gli Istituti Regionali per la Ricerca in campo educativo (IRRSAE), il Centro Europeo dell'Educazione a Frascati (CEDE) e la Biblioteca di Documentazione Pedagogica (BDP) a Firenze; inoltre furono date alle scuole delle norme per la sperimentazione e l'innovazione didattica, che rispecchiavano un impianto teorico-metodologico scientificamente ben impostato.

Si potrebbe affermare, con una certa fondatezza, che verso la metà degli anni settanta del XX secolo la ricerca empirica in campo educativo, nel nostro Paese, uscì dalla fase pionieristica ed ottenne il riconoscimento pubblico della sua rilevanza sociale anche perché in università le cattedre di Pedagogia sperimentale e di Docimologia iniziavano a non essere più delle 
«mosche bianche» e perché, oltre alle pubblicazioni di Calonghi e Visalberghi sulla sperimentazione, comparvero quelle di Becchi (1969), Gattullo (1970), Mencarelli (1970), Baratto (1970-1971), Trisciuzzi (1974), Inzodda (1975), Scilligo (1975) e Bertoldi (1976). Nello stesso periodo furono tradotti in lingua italiana diversi libri sulla sperimentazione, come quelli di Mialaret ${ }^{1}$ (1965), di Lovel e Lawson (1972) e di Dottrens (1973).

\section{L'HUMUS CULTURALE}

Il panorama pedagogico italiano nella prima metà degli anni cinquanta era in profonda mutazione. Continuava il dibattito, iniziato nel primo dopoguerra, tra la negazione della possibilità di una metodologia dell'insegnamento da parte degli eredi dell'attualismo gentiliano e l'esaltazione del metodo da parte del fisicalismo positivistico; ma in esso si inseriva la novità del progressivismo o attivismo dei nordamericani, che allora a molti sembrava inscindibile dalla sua matrice funzionalistica o strumentalistica. Queste tre correnti di pensiero si confrontavano in Italia con una secolare tradizione scolastica di ispirazione cristiana, che aveva saputo prendere da tutte le culture e da tutti i risultati della ricerca scientifica quello che potesse contribuire alla realizzazione integrale della persona umana nella sua pienezza. Il pensiero marxista non aveva ancora elaborato una proposta pedagogica organica anche se Gramsci aveva molto chiaro che il marxismo in Italia si doveva affermare per via culturale. Inizialmente i marxisti italiani furono diffidenti verso il pensiero deweyano, principalmente per la sua provenienza geografica; ma poi compresero che il suo primato dell'azione ben si coniugava con la loro ideologia.

Mentre il dibattito teorico si sviluppava lungo i filoni appena enunciati, la consapevolezza del ruolo dell'istruzione nella ricostruzione del Paese da parte di insegnanti e studiosi interessati all'ambito scolastico, produsse una serie di iniziative pedagogiche che crearono un humus favorevole alla sperimentazione scolastica. Senza pretesa di esaustività, ne ricordiamo alcune. L'istanza fondamentale dell'aderenza alla realtà del fanciullo e alle esperienze didattiche incentrate sulla valorizzazione dell'attività dell'alunno piuttosto che sul protagonismo dell'insegnante caratterizzò la linea editoriale della rivista toscana Puer, che fu attiva fra il 1950 e il 1960. Per i giovani insegnanti e per i docenti universitari che in quel decennio pubblicarono i loro contributi, l'aderenza alla realtà del fanciullo era un impegno: bisognava conoscere

1 Questo e gli altri testi di Gaston Mialaret ebbero una significativa diffusione in Italia a partire dalla metà degli anni settanta. 
meglio l'alunno per educarlo integralmente e non solo per favorire la sua integrazione sociale. Nel 1950 nacque la rivista Scuola e Città e nel 1954 iniziò la pubblicazione di Orientamenti Pedagogici. Le due riviste, promosse rispettivamente da Codignola e da alcuni docenti dell'Istituto Superiore Salesiano di Pedagogia, sia pure partendo da presupposti antropologici diversi, erano animate da un intento comune, la costruzione di un sapere pedagogico che si attenesse all'evidenza dei fatti educativi. Nel 1955 nacque Riforma della Scuola - diretta da L. Lombardo Radice, M.A. Manacorda, F. Zappa e C. Bernardini -, che voleva essere uno strumento di informazione e orientamento sulle questioni della scuola, considerate in rapporto con le questioni sociali ed economiche dal punto di vista marxista.

Anche De Bartolomeis contribuì a creare in Italia i presupposti per dare dignità scientifica alla ricerca empirica in campo educativo quando nel 1953, con il libro La pedagogia come scienza, presentò la Pedagogia come scienza pratico-poietica; successivamente sviluppò la sua attività nel promuovere nuove iniziative delle scuole e degli insegnanti piuttosto che condurre rigorose ricerche sperimentali. Il libro del 1969 La ricerca come antipedagogia documenta il tipo di evoluzione del suo pensiero in senso esperienziale.

Per la ricostruzione del clima che nel dopoguerra favorì la diffusione della ricerca empirica in campo educativo vale la pena considerare anche l'opera svolta dai tre Centri Didattici Nazionali per la diffusione e la valorizzazione delle migliori esperienze delle scuole elementari, delle scuole medie e dei licei; come pure l'azione del Movimento Circoli della Didattica e della sua rivista Ricerche Didattiche.

Infine, data la prevalenza in Italia di una pedagogia filosofica, non sono trascurabili le argomentazioni a sostegno della sperimentazione in pedagogia che negli anni cinquanta svolsero alcuni filosofi dell'educazione, di impostazione realista, come Casotti (1948), Braido (1952) e Calò (1957).

\section{LE CAUSE PROSSIME}

Tra la fine della seconda guerra mondiale e l'inizio degli anni cinquanta, tre furono gli eventi che, a mio modo di vedere, favorirono lo sviluppo della ricerca empirica in campo educativo in Italia: l'attrazione culturale degli USA che avevano vinto la guerra, la diffusione del pensiero di Dewey e la creazione dell'Istituto Superiore di Pedagogia da parte della congregazione salesiana.

Il contatto con le sperimentazioni scolastiche statunitensi faceva dire a Corallo (1955, p. IX), al suo ritorno da un soggiorno in USA durante l'intero anno scolastico 1952/53, quando visitò 80 scuole di 14 Stati: «Il 'metodo 
dell'esperienza', che ha trasformato la scuola americana in un immenso laboratorio sperimentale, non si è contenuto, com'è avvenuto altrove, nei limiti di un puro dilettantismo, ma si è inserito come parte integrante dello stesso programma e della 'filosofia' della scuola, che va aggiornando, di semestre in semestre, i suoi metodi e suoi programmi secondo i risultati dell'esperienza provocata e controllata. Questo sia detto senza voler obliare i difetti di questa stessa sperimentazione, lasciata spesso all'arbitrio di singole scuole o di singoli insegnanti e condotta anche in campi, già sufficientemente esplorati, dove la sua utilità è per lo meno discutibile». Nello stesso anno accademico anche Visalberghi svolse un soggiorno di studio negli Stati Uniti d'America.

La divulgazione del pensiero pedagogico di Dewey in Italia iniziò grazie alla pubblicazione delle sue opere per iniziativa di Lamberto Borghi, reduce dagli USA dove si era rifugiato per sfuggire alle persecuzioni razziali; grazie all'esposizione sistematica, in oltre 600 pagine, di tutte le opere del pedagogista americano fatta da Corallo nel 1950 quando in Italia di Dewey erano stati tradotti solo pochissimi lavori; grazie all'impegno di Aldo Visalberghi con il suo saggio su Dewey del 1951 e la precedente traduzione, nel 1949, di Logica. Teoria dell'indagine.

La lungimiranza dei superiori salesiani fece sì che, per sviluppare l'Istituto Superiore di Pedagogia, che poi divenne Ateneo e quindi Università Pontificia Salesiana, alcuni figli di don Bosco, tra cui Luigi Calonghi, fossero inviati a studiare le scienze dell'educazione nelle più prestigiose università internazionali.

\section{I PIONIERI}

Negli anni cinquanta del XX secolo l'orientamento sperimentale stentò a farsi strada nella ricerca pedagogica; all'inizio si trattò piuttosto di «Pedagogia esperienziale», per usare un'espressione mutuata da De Landsheere. Molto si deve a Borghi che promosse la traduzione italiana delle opere di Dewey, l'ispiratore della sperimentazione scolastica in USA; a De Bartolomeis che prestò molta attenzione alle esperienze degli insegnanti che intendevano riformare in senso attivistico la scuola italiana; all'azione di Ciari e del Movimento di Cooperazione Educativa che si ispirava a Freinet e Cousinet; ai «maestri sperimentatori» che si riunivano a Pietralba (BZ) per iniziativa di Agosti e Chizzolini per promuovere innovazioni didattiche con protocolli esperienziali. Senza dimenticare che l'approccio empirico ai fatti educativi o semplicemente didattici è rilevabile anche in altri ricercatori italiani degli anni cinquanta del secolo passato, è giusto segnalare che il passaggio dalla 
Pedagogia esperienziale alla Pedagogia sperimentale è avvenuto soprattutto per opera di Calonghi e Visalberghi perché seppero creare una scuola e delle strutture di ricerca. In Italia non erano mancate prima delle iniziative di Calonghi e Visalberghi, e in un certo senso anche di De Bartolomeis, esperienze scolastiche innovative che si possono classificare come Pedagogia esperienziale; molte di esse, ancorché prive della garanzia della rigorosità metodologica ebbero carattere sistematico e furono sottoposte a monitoraggio, ma i loro promotori erano animati meno da intenti scientifici e più da un'operosità innovativa in campo scolastico.

\section{Calonghi (I92 I-2005)}

Calonghi ha liberato l'approccio sperimentale allo studio dei fenomeni educativi dalla pregiudiziale empirista o neo-empirista; la sua diffidenza verso la cornice epistemologica empirista non gli ha impedito di sviluppare la dimensione empirica della ricerca sui fatti educativi. Calonghi ha dimostrato con i fatti di una mole impressionante di lavori scientifici (La Marca, 2007, pp. 126-151) che svolgere ricerca empirica in campo pedagogico-didattico non comporta la condivisione del quadro epistemologico del neo-empirismo. Anzi, subito dopo la sua prima opera di metodologia della ricerca, visti i pregiudizi esistenti tra gli studiosi, indipendentemente dalla loro fede religiosa, nei confronti del metodo sperimentale di ricerca in pedagogia, sentì l'esigenza di chiarire a tutti il suo punto di vista con un articolo comparso, nel 1957, su Orientamenti Pedagogici, "La sperimentazione e i suoi pericoli», dove critica chi ha una fiducia quasi fanatica nella sperimentazione e nello stesso tempo indica, insieme ai limiti, anche il contributo che il metodo sperimentale di ricerca può offrire al miglioramento delle prassi educative.

Calonghi fu fedele alla mentalità di don Bosco, che voleva conoscere bene i giovani, in un clima di amorevolezza, per educarli opportunamente ed era sempre pronto a modificare gli interventi educativi in base alle loro reazioni (Braido, 1997, pp. 71-72). Convinto che ci fosse continuità tra conoscenza empirica e conoscenza scientifica, Calonghi aiutò gli educatori a conoscere meglio $i$ giovani con strumenti validi e funzionali, li aiutò a verificare gli esiti delle loro azioni educative con procedure semplificate ma con fondamento scientifico.

I seminari filosofici organizzati dal siciliano Gemmellaro, che portavano gli studenti universitari a confronto diretto con i filosofi contemporanei avevano sviluppato in Calonghi una disposizione all'ascolto rispettoso di posizioni divergenti, che potevano tutte contenere qualche elemento da 
raccogliere, e l'attenzione per l'esercizio critico del pensiero nella ricerca della verità, senza opposizioni preconcette verso coloro che sostenevano posizioni diverse bensì con un atteggiamento di apertura cordiale verso chiunque fosse impegnato nella ricerca della verità. A 28 anni, dopo essersi laureato in Filosofia e in Teologia, Calonghi fu invitato dai suoi superiori a intraprendere un nuovo tipo di studi in Belgio, presso l'Università Cattolica di Lovanio.

Il contatto, negli anni accademici 1949/50 e 1950/51, con la scuola di Lovanio dove ricevette formazione universitaria in Pedagogia e Psicologia fece maturare l'interesse di Calonghi nei confronti dei metodi empirici per la ricerca in educazione. Per la sua tesi di laurea ebbe come relatore Raymond Buyse che era stato allievo di Decroly e con il quale aveva soggiornato in USA nel 1922. Per una strana coincidenza il primo corso di Pedagogia sperimentale fu affidato a Buyse a Lovanio nello stesso anno, 1923, in cui Mc Call pubblicava a New York How to experiment in education. Pertanto, quando Calonghi andò a studiare a Lovanio la ricerca sperimentale in campo educativo era coltivata in quella università da circa trenta anni.

L'incontro con R. Buyse indirizzò le scelte verso il rigore degli impianti di ricerca quantitativa, a cui però Calonghi sentiva l'esigenza di integrare strategie di ricerca qualitativa; in particolare approfondì la tecnica della riflessione parlata, che costituiva un valido strumento di rilevazione dei ragionamenti dei bambini nello svolgimento di compiti scolastici. Buyse aveva assunto da Claparède il metodo del pensare ad alta voce e l'aveva trasferito nella ricerca didattica per approfondire i meccanismi che stanno alla base degli errori e per conoscere quali vie i bambini usano spontaneamente nella risoluzione dei problemi che gli insegnanti propongono loro.

Tornato in Italia, dove l'idealismo era imperante, dovette ben presto affrontare le questioni epistemologiche legate all'introduzione del metodo sperimentale nella ricerca pedagogica; difese la proposta della ricerca empirica in campo educativo dalle accuse di rischio di determinismo implicito, di americanismo, di dipendenza dal positivismo e dal naturalismo. Mentre proponeva di assumere in campo educativo le strategie della sperimentazione, Calonghi dimostrava di avere consapevolezza dei limiti della scienza positiva dei fatti educativi; ma anche delle sue potenzialità di costruzione delle conoscenze pedagogiche a partire da fatti osservabili, delle possibilità che essa offriva di accumulare e socializzare dei fenomeni o eventi educativi corroborati dai dati ricavati dall'esperienza degli educatori.

Lo sforzo di Calonghi non venne subito compreso, anche da illustri studiosi. Per esempio, la rivista Archivio di Psicologia, Neurologia e Psichiatria, a p. 1162 del n. 6 del 1956, criticò con una veloce recensione anonima il primo manuale (Tests ed esperimenti. Metodologia della ricerca pedagogico-didattica), che Calonghi aveva pubblicato nel 1956 come frutto del suo biennio di stu- 
dio in Belgio e del successivo adattamento alla realtà italiana di quanto aveva appreso da Buyse e da Fauville. Alla recensione negativa Calonghi rispose subito (1957) e poi, per oltre un ventennio, dovette continuare a difendersi da chi non condivideva le sue scelte metodologiche per lo studio dei fatti educativi. Fu sostenuto nella sua difesa della sperimentazione in pedagogia dalle argomentazioni filosofiche di Braido e dall'influsso del realismo pedagogico di Casotti sui redattori dell'editrice "La Scuola» di Brescia, primo fra tutti Marco Agosti. Nell'argomentare sulla sperimentazione, per far capire a tutti che non era positivista, dovette dimostrare di conoscerne anche i pericoli, nel già citato articolo del 1957 pubblicato sulla rivista Orientamenti Pedagogici. Nell'adozione della ricerca positiva in campo pedagogico-didattico Calonghi si mostrava, fin dall'inizio, consapevole dei problemi epistemologici posti e attribuiva alla statistica, ai reattivi e agli schemi di sperimentazione una funzione strumentale; riteneva infatti che la tecnica, se applicata con flessibilità alle scienze umane, supplisce o rinforza la capacità conoscitiva del ricercatore, che è agevolato nel vedere globalmente la configurazione dei dati, nell'analizzarli, nel confrontarli e nel sintetizzarli.

Per fare accettare ai pedagogisti italiani, che in quel periodo erano per lo più filosofi dell'educazione, il suo nuovo modo di fare ricerca fu costretto a precisare la collocazione della Pedagogia sperimentale nel quadro epistemologico delle scienze dell'educazione, chiarendo che essa era solo una di tali scienze, senza alcuna pretesa di esaustività. Il dibattito, stimolante e fecondo, proseguì fino alla metà degli anni settanta (Calonghi, 1967, 1968, 1973, 1974, 1977).

Per ricostruire il clima in cui si svolse il dibattito iniziale basta ricordare che il terzo Convegno di Scholé, promosso nel 1956 dall'editrice «La Scuola» per i pedagogisti di ispirazione cattolica, ebbe per oggetto proprio la sperimentazione in campo educativo. Furono invitati a parlare anche Buyse e García Hoz (il fondatore della Pedagogia sperimentale in Spagna negli anni quaranta). I filosofi dell'educazione presenti misero in discussione le premesse che giustificano e stabiliscono i compiti e i limiti dell'indirizzo sperimentale in pedagogia. Fu in quella occasione che Corallo coniò l'espressione "prepedagogicità della sperimentazione», per far comprendere agli altri partecipanti al convegno che non si può fare un discorso scientifico sull'educazione senza aderenza ai fatti educativi empiricamente raccolti; secondo il pedagogista siciliano la sperimentazione offre infatti notizie indispensabili per la costruzione della metodologia dell'educazione, che egli denominava pedagogia speciale sottolineandone così la stretta unione con la pedagogia generale. Corallo riconosce quindi il valore della sperimentazione in campo educativo, le attribuisce il compito di preparare il discorso pedagogico, ma non ritiene che essa si possa qualificare come pedagogica. 
Altri ambienti culturali accolsero favorevolmente la proposta di Calonghi, come fece per esempio, nel 1957, la rivista Pedagogia e Vita recensendo il suo volume dell'anno precedente sulla metodologia della ricerca: è riconosciuto il rigore scientifico e lo spirito di ricerca dello studioso salesiano; si auspica che cadano i pregiudizi nei confronti di questo nuovo indirizzo dell'indagine pedagogica.

Per circa un ventennio, quando non insegnava ancora nell'Università statale ma solo in quella ecclesiastica dei Salesiani, Calonghi pubblicò alcune opere con cui si è formata un'intera generazione di sperimentatori in campo scolastico. Test ed esperimenti. Metodologia della ricerca pedagogico-didattica (1956) è stato il primo manuale italiano di guida alla sperimentazione pedagogico-didattica per studenti universitari e insegnanti; Elementi di metodologia della ricerca psicopedagogica (1956) riprende in forma divulgativa la materia del volume precedente; Dall'esperienza alla sperimentazione (1973) intende supportare sia chi sperimenta in forma rigorosa, sia l'insegnante che intende affinare la sua esperienza ordinaria; Sperimentazione nella scuola (1977) mette in evidenza come andrebbero rigorosamente pianificate, monitorate e verificate le innovazioni scolastiche, sia strutturali, sia didattiche, così da poterne vagliare i risultati, socializzarli e costruire conoscenza ed esperienza collettiva.

Molti anni dopo, nel 1993, nella presentazione del libro da lui curato Il bosco di Chirone. Contributi per l'identificazione della ricerca didattica, a p. 6 Calonghi scriveva: «Se si vuole uscire dall'incerto, dall'intuitivo, dall'approssimativo, dal frammentario bisogna lavorare a un sapere organico che si serve di un metodo sicuro e tipico per giungere a indicazioni di carattere operativo tali da guidare una crescita verso fini mirati, filosoficamente fondati ma visti poi nella concretezza operativa, rispettando situazioni descritte con rigore dalle scienze positive, sperimentali, seguendo ipotesi saggiate con una verifica autentica che ha stabilito la loro congruità a fini e situazioni». Siccome il libro costituisce il primo contributo collettivo dei docenti universitari fondatori della Società Italiana di Ricerca Didattica (SIRD), le parole introduttive del suo curatore fanno intravedere che in quel periodo il metodo sperimentale era stato già pienamente accolto in ambito accademico dagli studiosi della Didattica.

\section{VisAlbERGHi (I9I9-2007)}

Aldo Visalberghi ebbe come maestri durante gli studi universitari e postuniversitari Calogero, Bobbio e Codignola. Nel 1945 iniziò a lavorare come insegnante di Filosofia, Storia e Pedagogia nell'Istituto Magistrale di Aosta. 
Dal 1958 fu professore incaricato di Storia della Pedagogia all'Università di Torino e di Pedagogia in quella di Milano. Nel 1962 divenne professore ordinario di Pedagogia all'Università di Roma dove ha insegnato fino alla conclusione della carriera.

Nel 1949 Visalberghi tradusse una delle opere deweyane più impegnative e più dense di implicazioni per lo sviluppo della ricerca empirico-sperimentale, Logica, teoria dell'indagine, che era stata pubblicata a New York nel 1939. Il suo interesse per il pedagogista americano continuò a manifestarsi, attraverso la Rivista di Filosofia e la neonata rivista Scuola e Città, con recensioni delle sue opere, traduzioni di suoi brevi scritti e con la pubblicazione di saggi critici, che confluirono nella monografia J. Dewey del 1951. Nell'anno scolastico 1952/53, durante un soggiorno di studi negli Stati Uniti, all'età di 33 anni, usufruendo di una borsa di studio Fulbright, Visalberghi approfondì la sua conoscenza del pensiero di John Dewey e si accostò a quegli orientamenti di ricerca empirica che avevano subito in Italia l'ostracismo da parte della cultura idealista, in particolare al testing usato nella valutazione scolastica. Come è noto, Dewey non aveva condotto personalmente ricerche sperimentali nella scuola, ma ne era stato l'ispiratore.

Al pensiero di Dewey, in particolare alla sua opera Le fonti di una scienza dell'educazione (1928), si ispirava il saggio che Visalberghi scrisse nel 1958, Esperienza e valutazione, nel quale delineò un percorso che avrebbe potuto sviluppare anche in Italia la ricerca sperimentale in campo educativo. Il tema fu ripreso nel 1965 con il volume I problemi della ricerca pedagogica.

Intanto Visalberghi si era preoccupato di diffondere in Italia, come contemporaneamente faceva Calonghi, modelli e pratiche altrove già molto affermati, in particolare per quel che riguarda la valutazione e le sue tecniche: nel 1955 aveva pubblicato il volume Misurazione e valutazione nel processo educativo, col quale introduceva nella cultura educativa sensibilità e tecniche che erano ignorate o considerate con diffidenza.

Già come insegnante presso l'Istituto Magistrale di Aosta iniziò a collaborare con il Movimento di Cooperazione Educativa (MCE), che ispirandosi all'esperienza francese di Freinet, realizzava una pedagogia «esperienziale», vale a dire un tipo di ricerca che cercava di introdurre delle innovazioni scolastiche senza un controllo metodologico rigoroso, partendo da intuizioni di insegnanti riflessivi che si confrontavano tra loro. Già allora si nota l'attenzione di Visalberghi alla didattica "ludiforme» e collaborativa e alla "scuola aperta", che furono due costanti del suo pensiero pedagogico.

Successivamente l'attenzione di Visalberghi, come quella di altri pedagogisti politicamente vicini al socialismo o al comunismo, si concentrò sulla denuncia delle disuguaglianze sociali che la scuola contribuiva a riprodurre; concretamente, Visalberghi promosse indagini capaci di porre in evidenza le 
connessioni esistenti fra assetto sociale ed educazione scolastica. La raccolta di studi da lui curata, Educazione e condizionamento sociale (1964) denunciava con dati empirici scientificamente elaborati la selezione sociale avallata dalla selezione scolastica. In seguito dimostrò una notevole capacità di organizzare la ricerca empirica su tematiche educative e, in particolare, scolastiche promuovendo o partecipando a indagini nazionali e internazionali con grandi campioni di alunni e di insegnanti.

Intorno al 1970 Visalberghi aderì alla International Association for the Evaluation of Educational Achievement (IEA), un'associazione internazionale che era nata negli anni sessanta. Agli inizi degli anni settanta, mentre era diretta da De Landsheere, la IEA effettuò in un congruo numero di Paesi un'ampia indagine comparativa sull'apprendimento scolastico. La partecipazione italiana all'indagine del 1974 , preparata nei due anni precedenti, fu sostenuta in modo determinante da Visalberghi, malgrado la mancanza nel nostro Paese di strutture specificamente rivolte a sostenere la ricerca in campo scolastico.

I risultati della ricerca IEA segnalarono la presenza in Italia di tendenze negative che le ricerche condotte successivamente, fino alle più recenti rilevazioni OCSE-PISA, hanno confermato. In un certo senso si può ritenere simbolicamente il 1974, l'anno della prima indagine IEA a cui partecipò anche l'Italia grazie all'appassionato impulso di Aldo Visalberghi, il momento che segna l'uscita della ricerca empirica in campo scolastico dal periodo pionieristico e l'inizio di una fase che è tuttora in corso grazie al contributo degli allievi di Visalberghi e Calonghi e, fortunatamente, di tanti altri studiosi.

La nascita presso la "Sapienza - Università di Roma», nel 1983, del primo dottorato in Pedagogia sperimentale coordinato da Visalberghi, con la partecipazione di Calonghi, costituì un segno della consolidata accettazione accademica della ricerca empirica in campo educativo.

\section{UNA RIFLESSIONE FINALE}

La ricostruzione appena effettuata dell'appassionato lavoro svolto dai pedagogisti che hanno avviato la ricerca empirica in Italia mi induce a fare alcune considerazioni.

La ricerca empirica in campo educativo fornisce alla ricerca teorica indispensabili elementi di riflessione; essa consente di conoscere meglio la realtà educativa e di progettarne il miglioramento. Nella formulazione di una teoria pedagogica bisogna essere aderenti alla realtà osservabile negli alunni, negli educatori e nel contesto in cui si attua la relazione educativa. Certamente 
non si può ridurre il sapere pedagogico a ciò che è empiricamente osservabile nelle azioni degli educatori e dei giovani perché esso si alimenta anche dei risultati della ricerca filosofica, che indica qual è il bene possibile per l'uomo. Nello stesso tempo però non si possono dedurre i principi della metodologia educativa da principi filosofici assoluti, senza conoscere e osservare le persone da educare, i contesti e i processi. Le informazioni sulle problematiche educative, raccolte empiricamente, offrono un contributo essenziale alla costruzione dell'aspetto metodologico del sapere pedagogico che, oltre a individuare i fini dell'educazione, deve anche rispondere alla domanda su come si educa, non solo in generale ma anche in specifiche situazioni particolari.

Negli ultimi sessanta anni i ricercatori italiani hanno messo a punto numerosi metodi di ricerca empirica che, pur nella loro varietà e articolazione, si possono ricondurre alle due modalità classiche: l'osservazione sistematica e l'esperimento; il contributo di entrambe è imprescindibile per la costruzione delle metodologie educative.

Nella ricerca empirica si dovrebbe partire dalla rilevazione obiettiva delle pratiche educative per poi giungere a ricavare alcune costanti, dei principi orientativi per l'azione attraverso l'analisi delle correlazioni tra le variabili relative agli educatori, agli educandi e al contesto in cui si svolgono i processi educativi. Alcune correlazioni sono ipotizzate già nella fase progettuale mentre altre si scoprono in corso d'opera. La ricerca empirica di tipo osservativo si basa sulla fiducia nella capacità della mente umana di passare dall' "osservato» al «non osservato», vale a dire di effettuare un'inferenza induttiva. Il ragionamento induttivo presuppone che il campione di una realtà educativa esaminato dal ricercatore sia rappresentativo, con un certo grado di affidabilità, di tutte le situazioni che rientrano in quella particolare categoria di eventi educativi.

L'introduzione in un contesto dato, di una o più variabili ipotizzate come capaci di migliorare le relazioni educative costituisce il secondo modo di fare ricerca empirica, la sperimentazione. Si formulano ipotesi causali, la cui verifica richiede un impianto di ricerca più sofisticato di quello usato per la verifica delle ipotesi correlazionali. Quando si fa ricerca empirica di tipo sperimentale non si ha la pretesa di effettuare una generalizzazione dei risultati ottenuti, ci si accontenta - e, secondo me, non è poco - di affermare che in situazioni analoghe a quella in cui è stata condotta la ricerca si otterranno dei risultati simili. Ciò comporta che, oltre alla descrizione del fattore sperimentale e dei risultati ottenuti, si presti una particolare cura nel descrivere dettagliatamente il contesto in cui ha agito il fattore sperimentale o variabile indipendente. In questo modo si è in grado di affermare, ad esempio, che una certa innovazione educativa, positivamente sperimentata in un certo contesto, produrrà effetti ugualmente positivi in un contesto simile, con un determinato grado di probabilità. 
Mi sembra infine che facendo ricerca empirica, se essa vuole avere un valore per l'educazione, non basti porsi la domanda se la tecnica didattica sistematicamente osservata oppure intenzionalmente sperimentata produce un più efficace e pronto rendimento: occorre anche chiedersi se la tecnica che allevia il lavoro e lo facilita è anche educativamente, oltre che didatticamente, positiva.

\section{RIFERIMENTI BIBLIOGRAFICI}

Baratto, S. (1970-1971). Pedagogia sperimentale. Prima e seconda parte. Padova: Liviana. Becchi, E. (1969). Problemi di sperimentalismo educativo. Roma: Armando.

Bertoldi, F. (1976). Sperimentazione. Brescia: La Scuola.

Braido, P. (1952). Positivismo e positività in pedagogia. Salesianum, 3, 461.

Braido, P. (1997). Esperienza, osservazione, sperimentazione nella prevenzione educativa di don Bosco. In C. Nanni (a cura di), La ricerca pedagogico-didattica (pp. 71-83). Roma: LAS.

Calò, G. (1957). Intervento. In AA.VV., La sperimentazione in pedagogia, Atti del terzo Convegno di Scholé (pp. 92-106). Brescia: La Scuola.

Calonghi, L. (1956a). Elementi della metodologia della ricerca psicopedagogica. In AA.VV., Educare. Sommario di scienze pedagogiche (3 ${ }^{\mathrm{a}}$ ed., pp. 11-21). Zürich: PAS Verlag, 1963.

Calonghi, L. (1956b). Tests ed esperimenti. Metodologia della ricerca pedagogico-didattica. Torino: PAS (ultima ristampa, 1974).

Calonghi, L. (1957a). La sperimentazione e i suoi pericoli. Orientamenti Pedagogici, 4, 635-641.

Calonghi, L. (1957b). Tests ed esperimenti secondo l'indotta ignoranza d'un anonimo recensore. Orientamenti Pedagogici, 2, 255-259.

Calonghi, L. (1967). Esperienza, sperimentazione ed aggiornamento. Scuola di Base, 14(2-3), 68-81.

Calonghi, L. (1968). Piani di sperimentazione. Ricerche Didattiche 18(117-118), 218229 e 235.

Calonghi, L. (1973). Dall'esperienza alla sperimentazione. Scuola di Base, 20(2-3), 7-111.

Calonghi, L. (1974). La sperimentazione pedagogica. In M. Peretti (a cura di), Questioni di metodologia e didattica (pp. 723-744). Brescia: La Scuola.

Calonghi, L. (1977). Sperimentazione nella scuola. Roma: Armando (5a ristampa, 1989).

Calonghi, L. (a cura di). (1993). Nel bosco di Chirone. Contributi per l'identificazione della ricerca didattica. Napoli: Tecnodid. 
Casotti, M. (1948). Pedagogia generale, Vol. 2. Brescia: La Scuola.

Corallo, G. (1950). La pedagogia di Giovanni Dewey. Torino: SEI.

Corallo, G. (1955). Idee e fatti nelle scuole d'America. Salerno: Hermes.

De Bartolomeis, F. (1953). La pedagogia come scienza. Firenze: La Nuova Italia.

De Bartolomeis, F. (1969). La ricerca come antipedagogia. Milano: Feltrinelli.

De Landsheere, G. (1988). Storia della pedagogia sperimentale. Cento anni di ricerca educativa nel mondo. Roma: Armando (ed. orig., La recherche en éducation dans le monde. Paris: PUF, 1986).

Dewey, J. (1949). Logica. Teoria dell'indagine. Torino: Einaudi (ed. orig., Logic: The theory of inquiry. New York: Holt, Rinehart and Winston, 1938).

Dewey, J. (1951). Le fonti di una scienza dell'educazione. Firenze: La Nuova Italia. (ed. orig., The sources of a science of education. New York: Horace Liveright, 1929).

Dottrens, R. (1973). Pedagogia sperimentale e sperimentazione. Roma: Armando. (ed. orig., Qu'est-ce que la pédagogie expérimentale? Neuchatel: Delachaux et Niestlé, 1948).

Fiozzi, A. (1957). Recensione a L. Calonghi, Test ed esperimenti (Torino: PAS, 1956). Pedagogia e Vita, 18(4), 334-335.

Gattullo, M. (1970). Educazione e ricerca sperimentale: problemi e metodi. Bologna: Cooperativa Libraria Universitaria.

Inzodda, F. (1975). La ricerca pedagogica sperimentale. Brescia: La Scuola.

Laeng, M. (1997). La pedagogia sperimentale e la didattica sono discipline autonome? In C. Nanni (a cura di), La ricerca pedagogico-didattica (pp. 135-144). Roma: LAS.

La Marca, A. (a cura di). (2007). Ricerca, educazione, didattica. L'opera di Luigi Calonghi: sviluppi attuali. Palermo: Palumbo.

Lovel, K., \& Lawson, K. S. (1972). La ricerca in campo educativo. Presentazione di E. Becchi. Firenze: Giunti Barbera (ed. orig., Understanding research in education. London: University of London, 1970).

Mc Call, W. (1923). How to experiment in education. New York: McMillan Co.

Mencarelli, M. (1970). La sperimentazione nella ricerca pedagogica e nell'attività scolastica. Brescia: La Scuola.

Mialaret, G. (1965). Introduzione alla pedagogia sperimentale e sperimentazione. Torino: Loescher (ed. orig., 1963).

Scilligo, P. (1975). La sperimentazione nelle scienze dell'educazione. Torino: SEI.

Trisciuzzi, L. (1974). La ricerca sperimentale in pedagogia. Teramo: EIT.

Visalberghi, A. (1951). John Dewey. Firenze: La Nuova Italia.

Visalberghi, A. (1955). Misurazione e valutazione nel processo educativo. Milano: Edizioni di Comunità. 
Visalberghi, A. (1958). Esperienza e valutazione. Torino: Taylor.

Visalberghi, A. (a cura di). (1964). Educazione e condizionamento sociale. Roma - Bari: Laterza.

Visalberghi, A. (1965). I problemi della ricerca pedagogica. Firenze: La Nuova Italia.

\section{RiASSUNTO}

Mentre la filosofia dominante in Italia alla fine della seconda guerra mondiale non era favorevole alla sperimentazione pedagogica, alcune iniziative, tese a innovare in senso attivistico i contenuti e $i$ metodi dell'insegnamento scolastico, costituirono le cause remote dell'avvio dello studio dell'educazione con il metodo positivo. Esse facilitarono l'azione dei pionieri della ricerca empirica in campo educativo, che si sviluppò in Italia a partire dagli anni cinquanta del XX secolo per tre cause prossime: l'influsso delle sperimentazioni realizzate nelle scuole degli USA, la diffusione del pensiero di Dewey, l'impulso dato dall'stituto Superiore di Pedagogia dei Salesiani allo studio delle scienze dell'educazione. Calonghi e Visalberghi, per primi, riuscirono a promuovere e a organizzare due scuole di ricerca empirica sui fatti educativi che hanno contribuito al pieno riconoscimento scientifico della Pedagogia sperimentale e della Docimologia a metà degli anni settanta. Per evitare di cadere nel tecnicismo, i ricercatori che usano il metodo sperimentale avvertono l'esigenza di inquadrare i loro studi specialistici all'interno di un vasto orizzonte di senso pedagogico.

Parole chiave: Calonghi, Pedagogia esperienziale, Pedagogia sperimentale, Ricerca empirica, Visalberghi. 